\title{
Acute myeloid leukemia under lenalidomide therapy in a patient with multiple myeloma
}

\author{
Ali Eser ${ }^{1}$, Funda Pepedil Tanrikulu ${ }^{2 *}$, Ayse Tulin Firatli Tuglular ${ }^{1}$
}

\begin{abstract}
A 53-year-old patient who had a 7 year history of multiple myeloma was diagnosed with high risk myelodysplastic syndrome while on lenalidomide treatment. Progression to acute myeloid leukemia has occurred during the following 4 months. Like our case, secondary primary malignancies (SPMs) can rarely complicate treatment of multiple myeloma. Although possible association between SPMs in patients receiving lenalidomide can be attributed to previous melphalan use, potential SPM risk with lenalidomide cannot still be ruled out safely
\end{abstract}

Key words: Multiple myeloma; Lenalidomide; Acute myeloid leukemia

\section{Introduction}

A 53-year-old woman was diagnosed with multiple myeloma (MM) in 2007. Autologous stem cell transplantation (ASCT) was performed after achieving complete remission (CR) with 4 cycles of vincristinedoxorubicin-dexamethasone (VAD) therapy. The patient was in CR after ASCT. The disease relapsed in 2012. Bortezomib and dexamethasone was initiated and partial remission was achieved after four cycles. Second ASCT was performed and the patient was followed up in $\mathrm{CR}$ for 2 years. Clinical relapse was observed within two years after the ASCT, treatment with lenalidomide and dexamethasone was initiated. During the fourth cycle, grade IV neutropenia, anemia, and thrombocytopenia was observed. The treatment was discontinued due to severe pancytopenia requiring routine transfusions. A bone marrow aspiration and biopsy was performed. Significant dysmorphic alterations in megakaryocytes, myeloid and erythroid cell lines were evident. Eight percent of all bone marrow cells were myeloblasts.

Clonal plasma cells were not observed. She was diagnosed with myelodysplastic syndrome, refractory anemia with excess blasts type I (MDS-RAEB1) and azacytidine treatment was initiated.

During the second cycle of azacytidine, patient was hospitalized for intravenous antibiotic treatment due to bacterial pneumonia. Azacytidine was interrupted for approximately two months. A repeat biopsy was performed, which revealed 53\% myeloblasts consistent with a diagnosis of acute myeloid leukemia (AML).
Induction chemotherapy with idarubicin for three days and cytarabine for seven days were commenced. Bone marrow biopsy on the 28th day showed $13 \%$ myeloblasts. Patient died before salvage chemotherapy due to septicemia.

\section{Discussion}

In multiple myeloma, SPM incidence is about $6.1 \%$ per year in twenty years being comparable to expected incidence in general population. There is a correlation between long-term melphalan use and AML/MDS development (1).

According to the lenalidomide maintenance trial reported by Attal et al, SPM incidence in lenalidomide arm was 3.1 and 1.2 in placebo arm for every 100 patient years $(p=0.002)$. Thirteen hematological malignancies in lenalidomide arm and 5 in placebo arm were reported. However number of AML and MDS cases was similar (2).

Similiarly, cumulative SPM incidence in the study reported by Mccarthy et al was $8 \%$ in lenalidomide arm and $3 \%$ in placebo arm. Eight cases of hematological malignancies were detected in lenalidomide arm and six of these eight cases were MDS or AML. Only one hematological malignancy was detected in placebo arm (3). In another literature by Palumbo et al. 10 cases of MDS or AML were reported in lenalidomide arm corresponding to an incidence of $2.6 \%$. (4). 
Table: Incidence of SPMs with lenalidomide according to the reported literature

\begin{tabular}{lclccc} 
Reference & $\begin{array}{c}\text { Total } \\
\text { number of } \\
\text { patients }\end{array}$ & $\begin{array}{c}\text { Randomised } \\
\text { arms }\end{array}$ & $\begin{array}{c}\text { Number of } \\
\text { invasive } \\
\text { SPMs }\end{array}$ & $\begin{array}{c}\text { Number of } \\
\text { hematologic } \\
\text { malignancies }\end{array}$ & $\begin{array}{c}\text { Number } \\
\text { of MDS or } \\
\text { AML }\end{array}$ \\
\hline Attal et al. (2) & 614 & Lenalidomide & 23 & 13 & 5 \\
& & Placebo & 9 & 5 & 4 \\
McCarthy et al. (3) & 460 & Lenalidomide & 18 & 8 & 6 \\
Palumbo et al. (4) & \multirow{2}{*}{459} & Placebo & 6 & 1 & 0 \\
& & MPR-R* & 12 & 5 & 5 \\
& & MPR & 9 & 1 & 5 \\
\hline
\end{tabular}

* MPR-R: melphalan-prednisone-lenalidomide induction followed by lenalidomide maintenance, MPR: melphalan-prednisone-lenalidomide, MP: melphalan-prednisone

However, in all of these above mentioned literature which were also presented in the table, lenalidomide was incorporated into the induction with a melphalanbased chemotherapy, either used before ASCT, or given as maintenance therapy after ASCT or melphalan-based chemotherapy. Various studies reported safety data for lenalidomide regarding SPM incidence $(5,6)$. The incidence of SPM is about 1.5$7.4 \%$, which is comparable to SPM incidence in healthy population.

\section{Conclusion}

Although possible association between SPMs in patients receiving lenalidomide can be attributed to previous melphalan use, potential SPM risk with lenalidomide cannot still be ruled out safely.

Conflict of Interest: The authors declare no potential conflicts of interest with respect to the research, authorship, and/or publication of this article.

\section{References}

1. Kyle RA, Pierre RV, Bayrd ED. Multiple myeloma and acute myelomonocytic leukemia. $\mathrm{N}$ Engl $\mathrm{J}$ Med. 1970;283(21):1121-5.

2. Attal M, Lauwers-Cances V, Marit G, Caillot D, Moreau P, Facon T, et al. Lenalidomide maintenance after stem-cell transplantation for multiple myeloma. N Engl J Med. 2012;366(19):1782-91.

3. McCarthy PL, Owzar K, Hofmeister CC, Hurd DD, Hassoun H, Richardson PG, et al. Lenalidomide after stemcell transplantation for multiple myeloma. N Engl J Med. 2012;366(19):1770-81

4. Palumbo A, Hajek R, Delforge M, Kropff M, Petrucci MT, Catalano J, et al. Continuous lenalidomide treatment for newly diagnosed multiple myeloma. $\mathrm{N}$ Engl J Med. 2012;366(19):1759-69.

5. Dimopoulos MA, Richardson PG, Brandenburg N, Yu Z, Weber DM, Niesvizky R, et al. A review of second primary malignancy in patients with relapsed or refractory multiple myeloma treated with lenalidomide. Blood. 2012;119(12):2764-7.[]

6. Rossi AC, Mark TM, Jayabalan D, Christos PJ, Zafar F, Pekle K, et al. Incidence of second primary malignancies (SPM) after 6-years follow-up of continuous lenalidomide in first-line treatment of multiple myeloma (MM). J Clin Oncol. 2011;29: abstr 8008

Copyright $(2014$ The Author(s); This is an open-access article distributed under the terms of the Creative Commons Attribution License (http://creativecommons.org/licenses/by/4.0), which permits unrestricted use, distribution, and reproduction in any medium, provided the original work is properly cited. All Rights reserved by international journal of Medical Science and Discovery. 